Commentary on: Inflexibly Enacted Traditional Masculinity Norms (IE-TMNs) and Their Impact on Adolescent and Young Adult Depression: The Hybrid Case Study of "Tommy"

\title{
Understanding the Role of Inflexibly Enacted Masculinity Norms in Therapy: A Commentary on the Case of "Tommy"
}

\author{
JAMES R. MAHALIK ${ }^{\mathrm{a}, \mathrm{b}}$ \\ ${ }^{\mathrm{a}}$ Boston College \\ ${ }^{b}$ Correspondence regarding this article should be sent to: James R. Mahalik, Champion Hall 312, Boston College, \\ Chestnut Hill MA 02467 \\ Email: Mahalik@bc.edu
}

\begin{abstract}
The case of "Tommy" highlights both an underutilized methodology for increasing the field's understanding of the therapeutic process in working with men, and the importance of addressing inflexibly enacted masculinity norms. My commentary focuses on detailing how the methodology helps those of us interested in understanding how masculine norms contribute to men's presenting concerns, help-seeking, and experiences in treatment focusing on the multidirectionality of relational experiences, masculine identity development, and presenting issues. Then I discuss how the concepts of inflexibility has its roots within an Interpersonal Therapy framework (e.g., Kiesler, 1983) and how this inflexibility develops consistently with explanations contained in Precarious Manhood Theory (i.e., Vandello \& Bosson, 2013).
\end{abstract}

Key words: psychology of men and masculinity; adolescents; masculine identity development; inflexiblyenacted traditional masculine norms (IE-TMNs); interpersonal therapy; precarious manhood theory; case study; hybrid case study

\section{METHODOLOGICAL CONSIDERATIONS}

I appreciate the opportunity to comment on Dr. Christopher Dewey's (2020) case study of Tommy as it represents an under-utilized research methodology within the psychology of men and masculinities (Mahalik, 2014), and it offers the promise of making a significant contribution to our understanding of clinical applications of masculinity issues in working with men. I say this because the study of men and masculinities has largely been dominated by quantitative survey studies without detailed clinical context in addressing issues such as help-seeking (see Seidler et al., 2016, for a review), men's presenting concerns (Wong et al., 2017), and experiences men have around treatment (Mahalik et al., 2012). Because of this our field does not often have an illustration of how those concepts play out in clinical work with actual clients. I see the case study methodology utilized in Dr. Dewey's study as not only having stronger external validity than more traditional research designs in the psychology of men and masculinities, but also as 
illustrating the dynamics of gender for Tommy as he approaches getting help, and understanding both his presenting problems, as well as his experiences in treatment. For example, men's helpseeking is often characterized as "men are reluctant to get help", "men are particularly reluctant to get help for mental health issues", and "men who conform to traditional masculine norms are the most reluctant to get help for mental health issues." What the reader usually misses in this type of research is how help-seeking is situated within the contextual experiences of personal history, relational difficulties, psychological distress, and the specific cultural mandates that place barriers to getting help for mental health concerns. In the case presented by Dr. Dewey, the reader is able to see this context such that we can appreciate how help-seeking is connected to Tommy's struggles with vulnerability and emotional disclosure and draws attention to the therapist's work of paying attention to the client's comfort zone. We get a good sense from the study about how seeking help and staying in treatment for the therapist's male clients is connected to the timing and pace of exploration into painful feelings and internalized beliefs about masculinity that are learned in familial, interpersonal, and institutional contexts.

The usual research in the psychology of men and masculinity also consistently finds conformity to traditional masculine norms as being related to an array of presenting concerns including many that Tommy presents with in the case such as substance abuse, depression, relational and intimacy concerns. However, the case study results help the reader understand that these presenting concerns are not distinct, orthogonal presenting issues. Rather, Tommy's relational concerns, family dynamics with male and female family members, experiences of depression, problematic experiences with women, episodes of school discipline from alcohol use all intersect and affect each other. The case study illuminates that these both form Tommy's identification with normative male behavior and are also expressions of that normative male behavior. For example, his relational experiences lead him to reach certain conclusions about what it means to be a man (e.g., being a failure is shameful), that sense of normative male behavior then leads to actions that contribute to his presenting concerns (e.g., substance abuse in response to feelings of shame and depression), and the resultant negative consequences that occur through those actions (e.g., disciplinary actions from the school because of harassment). In doing so, Dr. Dewey illustrates the multi-directional influence of relational experiences, masculine identity development, and presenting issues which goes beyond the usual understanding we have of the relationship between conformity to masculine norms and presenting concerns in traditional research designs.

The case study also gives us insight into Tommy's experiences with treatment that are significantly more insightful than the usual outcomes used in the evaluation of treatment effectiveness or whether Tommy terminated treatment "early." Indeed, some of the most illustrative lessons from the case study findings that would never surface in traditional psychotherapy outcome research is how the therapist progressively moves the focus of sessions from external events such as university discipline to more of a focus on personal behaviors such as alcohol use to then move more into his emotional life by exploring his depression. This progression of the work with the therapist over time provides a much richer understanding of therapeutic change than the usual change scores reported in randomized clinical trials. 
It was also a strength, though, to utilize well-established outcome measures from more traditional evaluation research such as the Outcome Questionnaire-45 (OQ-45) and Patient Health Questionnaire-9 (PHQ-9). The integration of this information into the case study helped the reader have a sense of how Tommy's case might look in terms of traditional evaluation of treatment, along with the narrative of what was happening in sessions and Tommy's own descriptions of how he was feeling, acting, and relating in his life. The use of the Meaning of Adolescent Masculinity Scale (MAMS) was a useful measure so that both Tommy and his therapist could have a tool to use for exploring salient masculine norms for the client. Because men are often less aware of themselves as gendered persons, I believe that masculinity measures such as the MAMS can be a useful way to concretely explore of male gender-role issues and their effects into therapeutic work (e.g., Mahalik et al., 2005).

As the study of masculinities is currently burgeoning with new constructs and innovative measures for assessing them, clinicians may consider learning about these constructs and adopting other measures to help male clients explore how issues of gender contribute to their presenting concerns, as well as their experiences of the treatment process. For example, the Subjective Masculinities Stress Scale (Wong et al., 2013) is an example of an individually tailored assessment tool that can help men explore their personal experiences of what it means to be a man. If Tommy were to rate the item "As a man, I need to be a success in life" as "almost always/always" stressful, this could lead to him being invited to explore the experiences of failure or perceived failure, their connections to problematic actions or coping responses, and the socialization influences (e.g., parents, coaches, peers) that communicated to that being a man means being successful in life.

Thus, I believe the case study methodology presented by Dr. Dewey makes some significant contributions to understanding and illustrating how personal and family history, relational difficulties, and the cultural mandates around gender roles are mutually influencing and contribute to our understanding of client help-seeking, presenting concerns, and experiences in treatment. Of course, there are also significant limitations to the case study method including the limited generalizability of the findings from this case to the experiences of living and therapy for other groups of men. It is notable that the case of Tommy was compiled as a composite from other, presumably, White, heterosexual, educated, cis-gendered men. This is a significant limitation to making statements about the therapeutic implications of the case for other men than Tommy as our understanding of how masculinities operate in treatment related to issues of depression, alcohol abuse, conformity to masculine norms, help-seeking, and the progression of therapy are all likely to vary as these intersect with age, race, gender identity, immigration status, language, sexual orientation, class, religion, and other social, political, and personal variables.

\section{THEORETICAL CONSIDERATIONS}

In addition to the methodological contributions of the paper, Dr. Dewey also draws the reader to consider the importance of the central construct explored in the case study, namely inflexibly-enacted traditional masculine norms (IE-TMNs). In centering this concept in his analysis, Dr. Dewey highlights what I view as the most shared understanding of psychopathology across all clinical therapeutic models. Namely, flexibility is the hallmark of 
health and well-being and rigidity is the common feature of pathology and interpersonal distress. In working with Tommy, Dr. Dewey utilizes a cognitive-behavioral approach which is very well received by Tommy and consistent with recommendations by persons in the field that traditionally masculine men may find the directive approach to be more comfortable (Brooks, 2010). Having said that, I think the focus on inflexibly-enacted traditional masculine norms (IETMNs) necessitates a recognition that these ideas emerge from the work of Interpersonal Psychotherapy (Leary, 1957), especially Kiesler's model of Interpersonal Theory (Kiesler, 1983). In this model, the client who experiences difficulties "consistently broadcasts a rigid and extreme self-presentation and simultaneously pulls for a rigid and constricted relationship from others" (Kiesler, 1988, p. 17). The thinking from the case study is that socializing boys and men into traditional masculine roles is not problematic, per se, but their enactment of these roles in a rigid way is what causes problems for the individual, his family and groups to which he belongs (e.g., work), and his broader community and society (see Mahalik, 1999).

For example, boys and men are often socialized to control their emotions through suppressing vulnerable feelings. Although emotional expression and intimacy are critical parts of human well-being, having the ability to control vulnerable feelings can be situationally very adaptive. You would not want your fire-fighter or surgeon to be overcome by fear or sadness if they were rescuing you from a burning building or performing life-saving surgery on you. Similarly, boys and men are typically socialized to be self-reliant and independent. Although interdependence is a central part of the human experience, being able to solve one's own problems and be able to make one's way in the world without being over-reliant on others are admirable qualities. Difficulties arise, however, when men are unable to bring any other interpersonal behaviors to their relationships or interactions with others. When relationships call for intimacy and vulnerability, when a boy or man is suffering physical or emotional distress, or when someone is struggling to cope with a problem and should seek help, from the perspective of interpersonal psychotherapy, only being able to respond to that situation with emotional detachment and hyper-independence is what is seen as pathological in the model. Indeed, research finds that the more extreme men are in their enactment of traditional masculine norms, the more likely they are to report negative affect, less likely to report positive affect, and less likely to seek help (Wong et al., 2017).

Having said that it is this rigid enactment of masculine norms that is problematic, not the masculine norms themselves, I also want to be clear that there are some norms communicated to men that reflect what Pleck (1995) labels as "dysfunction strain." That is, certain masculine norms are not problematic only when enacted in inflexible ways (as illustrated in this case), but are inherently problematic. These are norms that communicate that men's power should be enacted without consideration to the harmful effects on others and is most often seen related to violence, misogyny or exercising power over women, and the type of disdain for sexual minorities that creates harm.

Returning to the case, we see how Tommy's inflexible enactment of masculinity norms is the source of the problem. For example,

in reaction to these negative thoughts and feelings, Tommy's enactment of stereotypically masculine behaviors became more rigid. He was more likely to become angry and 
aggressive, he sought out hookups with female peers to regain feelings of attractiveness and sexual desirability, and he increased his alcohol intake in an attempt to dampen negative feelings, reduce social inhibitions, and express himself more openly (Dewey, 2020, p. 257).

What we see in the case is an example of the detached and dominant interpersonal style that was reinforced by societal messages that encourage boys and men to follow the (1) "no sissy stuff," (2) "big wheel," (3) "give "em hell," and (4) "sturdy oak" injunctions of masculinity that David and Brannon (1976) describe in some of the earliest articulation of masculine norms. These injunctions are associated, respectively, with messages to men that they (1) should avoid anything feminine (e.g., being dependent or vulnerable), (2) should strive to defeat others and achieve status by getting to the top, (3) should actively seek out adventure and risk, responding with violence if necessary, and (4) should never show weakness by enduring difficulties without relying on others for help. This detached and dominant interpersonal pattern typically leads to difficulties broadly around issues with intimacy and connection, as well as issues connected to power such as aggressiveness, including sexual aggressiveness, anger management, power over women, and fear of inferiority or failure. It is not coincidental that we see all of these issues in the case of Tommy, which is a compilation of issues across Dr. Dewey's clinical experience of working clinically with men.

Given these types of issues associated with the rigid enactment of detached and dominant interpersonal styles, therapists can expect that these rigid patterns will leave male clients feeling more isolated and lonely with less hope of things changing. Again, we see these dynamics as occurring in the case of Tommy when Dr. Dewey writes,

Although his drinking temporarily allowed him to feel free, engage in fleeting sexual encounters, and avoid negative thoughts about himself, he often felt more depressed in the aftermath. This pattern of behavior made it harder for him to attend his classes and maintain his grades, and his friends soon became irritated by his erratic actions. On top of that, he was getting into trouble and putting his enrollment status at risk. Failing to live up to his beliefs about masculinity, Tommy grew increasingly depressed and continued to drink, creating a cycle that prevented him from making meaningful changes in his life and further isolated him (Dewey, 2020, p. 257).

From an interpersonal psychotherapy framework, "the goal of therapy is to facilitate an increased frequency and intensity of interpersonal acts with significant others from segments on the Circle opposite those that define the patient's pattern of maladaptive interpersonal behavior" (Kiesler, 1988, p. 23). Thus, in the case of Tommy, the therapeutic goals are to reduce the intensity of his interpersonal behaviors in this detached-dominant interpersonal style and promote access to other interpersonal behaviors that promote connection and mutuality.

As Interpersonal Theory is useful for helping us to better understand the case of Tommy in relation to the central construct in the case analysis (i.e., inflexibly enacted masculinity norms), one might also reasonably ask "from where does this inflexibility come from?" To help answer that question, I want to introduce the reader to a second theoretical framework that may be useful to consider, namely Vandello and Bosson's model of Precarious Manhood. They help explain the development of rigid conformity to masculine norms by positing that manhood is 
understood "as a precarious social status that is hard won and easily lost, and that requires continual public demonstrations of proof" (p. 101, Vandello \& Bosson, 2013). Thus, men must remain vigilant of "social transgressions and shortcomings" that might jeopardize their manhood status (p. 28; Bosson \& Vandello, 2011).

In the case we can see examples of Tommy's experiences of feeling the loss of status in a number of experiences in relationships and with the disciplinary outcomes. In response to the feeling of threat from these experiences of loss of masculine status, men would be more likely to overconform to masculine norms to try to re-establish masculine status. There are a number of illustrations of this in the case of Tommy, particularly in his loss of masculine status being romantically rejected and then the humiliation of being disciplined. In response to losing status in these events his adoption of the hard-drinking man overconforms to masculine norms around drinking with Dr. Dewey reporting that Tommy also spoke about how drinking gave him an excuse to be more "wild" than he usually was and exhibit a sense of fearlessness, becoming the angry man in that he had "become irritable, aggressive, and nasty on a few occasions because of drinking and had upset many of his friends, ... [and that he had been] written up for harassing a female peer at her dorm after drinking” (p. 265).

In this example, we see Tommy over-conforming to the hard-drinking, aggressive guy, who wants to establish his dominance over someone who is rejecting him when his is faced with rejection and the loss of masculine status.

In conclusion, I think the case study makes two meaningful contributions to the literature on therapeutic work with men. First, the methodology provides a richer understanding of the contextual dynamics that are part of the development of problems and effect on both helpseeking and the experience of therapy. Second, it highlights the critical idea of masculinity norms as problematic due to their inflexibility, which is founded in Interpersonal theory and explained by the Precarious Manhood model.

\section{REFERENCES}

Brooks, G. R. (2010). A transtheoretical model. In G. R. Brooks (Ed.), Beyond the crisis of masculinity: A transtheoretical model for male-friendly therapy (pp. 139-169). Washington, DC: American Psychological Association.

Dewey, C. (2020). Inflexibly enacted traditional masculinity norms (IE-TMNs) and their impact on adolescent and young adult depression: The hybrid case study of "Tommy."

Pragmatic Case Studies in Psychotherapy, 16 (3), Article 1, 237-304. Available: http://pcsp.libraries.rutgers.edu/

Kiesler, D. J. (1983). The 1982 interpersonal circle: A taxonomy for complementarity in human transactions. Psychological Review, 90, 185-214.

Leary, T. (1957). Interpersonal diagnosis of personality. New York: Ronald.

Mahalik, J. R. (2014). Both/and, not either/or: A call for methodological pluralism in research on masculinity. Psychology of Men and Masculinity, 15, 365-368.

Mahalik, J. R. (1999). Interpersonal psychotherapy with men who experience gender role conflict. Professional Psychology: Research and Practice, 30, 5-13. 
Mahalik, J. R., Good, G. E., Tager, D. T., Levant, R. F., \& Mackowiak, C. (2012). Developing a taxonomy of helpful and harmful practices for clinical work with boys and men. Journal of Counseling Psychology, 59, 591-603.

Mahalik, J. R., Talmudge, W. T., Locke, B. D., \& Scott, R. P. J. (2005). Using the Conformity to Masculine Norms Inventory to work with men in a clinical setting. Journal of Clinical Psychology, 61, 661-674.

Seidler, Z.E., Rice, S.M., Ogrodniczuk, J.S., Oliffe, J.L., Shaw, J.M., \& Dhillon, H.M. (2019). Men, masculinities, depression: Implications for mental health services from a delphi expert consensus study. Professional Psychology: Research and Practice, 50, 51-61.

Vandello, J. A., \& Bosson, J. K. (2013). Hard won and easily lost: A review and synthesis of research on precarious manhood. Psychology of Men \& Masculinity, 14, 101-113.

Wong, Y.J., Ho, M.R., Wang, S., \& Miller, S.K. (2017). Meta-analyses of the relationship between conformity to masculine norms and mental health-related outcomes. Journal of Counseling Psychology, 64, 80-93. 